\title{
An estimate of chronic disease burden and some economic consequences among the elderly Hong Kong population
}

\author{
J Woo, S C Ho, S G Chan, A L M Yu, Y K Yuen, J Lau
}

\begin{abstract}
Objectives-To estimate the burden of chronic disease for an elderly Chinese population aged 70 years and over, and to illustrate the use of this information in estimating the economic consequences of disease burden using stroke as an example.

Participants-A total of 1902 subjects recruited by random sampling of the old age and disability allowance schemes, which cover over $90 \%$ of the Hong Kong elderly population, stratified by sex and five year age groups from age 70 years onwards.
\end{abstract}

Method-Information was collected on 10 medical conditions at baseline: arthritis, hypertension, cardiac disease, stroke, chronic obstructive airways disease, peptic ulcer, diabetes mellitus, osteoporotic fracture, malignancy, and dementia. A follow up survey was carried out after 18 months to determine the occurrence of new disease and the number with disease who had died. Disease burden is calculated as the number with disease at baseline plus the number developing new disease minus the number who had died.

Results-Disease burden figures were highest for arthritis, hypertension, cardiac disease, and peptic ulcer, and were higher in the 70-79 age group than the 80+ age group for some diseases. For stroke, the economic cost based on a population projection for 2001 was estimated to be around HK\$1 900000000 , or US\$250 million.

Conclusion-Information on the burden of chronic disease is important. It enables the economic consequences to be estimated so that strategies can be developed to prevent diseases with high costs and known effective preventive methods.

(F Epidemiol Community Health 1997;51:486-489)

In any country, the elderly population consumes a large proportion of health care resources. In part, this is a result of the increasing prevalence of chronic diseases with age. Information regarding chronic disease burden in a population is of economic importance since it is needed for estimating resource needs for treating particular diseases, and also for estimating the economic effects of reducing the incidence of certain diseases as a result of population prevention efforts. Information on the occurrence of chronic diseases in the elderly is usually in the form of prevalence or incidence rates from cross sectional and prospective studies. However, from the resource utilisation point of view, it may be more relevant to consider the disease burden, which represents the pool of disease to which there is a constant addition (from development of disease in the previously well population) and elimination (as a result of death of individuals with the disease). Estimation of disease burden would take into account the incidence of the disease over a defined period, the point prevalence at baseline, and death during the same period among those with the disease. In order to provide such an estimate, we used data obtained from a cross sectional and prospective health and social survey of elderly Chinese aged 70 years in Hong Kong to calculate the burden for 10 diseases. The economic consequence is also estimated for one disease (cerebrovascular disease) as an example of the usefulness of such data.

\section{Methods}

The survey subjects were recruited by random sampling of the old age and disability allowances schemes, stratified by sex and five year age groups (70-74, 75-79, 80-84, 85-89, 90+). The old age allowance scheme is a non-means tested scheme; anyone aged 70 years and over and a resident of Hong Kong for five years is eligible to enrol in it. It covers over $90 \%$ of the Hong Kong elderly subjects and has been utilised for recruitment of subjects in a previous study on the elderly. ${ }^{1}$ The normal disability and higher disability allowance schemes provide the disabled with further financial assistance. The latter is twice the amount of the former and is meant for the severely disabled who require constant attendance from others in their daily life. Together, all these schemes provide territory-wide coverage of over $90 \%$ of the Hong Kong elderly. Those not on the schemes are likely to be the extremely rich.

For the old-old group (aged 80 years and above), 150 subjects were drawn from each of the six strata, male and female by age groups $80-84,85-89,90+$, so the total old-old sample consisted of 900 subjects. A sample size ${ }^{\circ}$ of 150 in each of the cells allows the detection of a difference of $15 \%$ between the groups with a power of at least $70 \%$ and at the $5 \%$ level of significance. For the 70-79 years age group, 300 subjects were drawn from each of the four 
strata-male and female by age groups $70-74$ and 75-79. With the assistance of the Social Welfare Department, letters of invitation to participate in the study were sent to the sampled subjects. Letters were sent in batches until the required number in each age group had been obtained. Sixty per cent of the subjects reached gave their consent. The response rate was higher in the $80+$ age group compared with the 70-79 age group, suggesting perhaps that the non-responders may have been more fit.

Data collection was done through both personal interview and physical assessment of the respondents. All interviews were conducted at the subjects' place of residence. Personal interview was done using a structured questionnaire. This was based on a number of developed and validated instruments from overseas, but these have been tested and validated in the local setting. The instruments included functional assessment using the Barthel index, ${ }^{2}$ psychological assessment using the geriatric depression scale $^{34}$ and the information/orientation section of the Clifton assessment procedure for the elderly, ${ }^{5}$ and symptom assessment (chest pain, dyspnoea, cough) using the WHO angina and dyspnoea questionnaires. $^{6}$ Not all the information collected was used for this report. The questionnaire was translated into spoken Cantonese to minimise variations between interviewers. An instruction manual was designed to accompany the interview schedule.

A pilot study was done during February to March 1991. Fifty subjects from both the community and institutions were included in the pilot study. Modifications of the questions and assessment were done as a result of the pilot study. The survey proper was carried out during the period of July 1991-July 1992. Data collection was done by 31 registered nurses and interviewers with a medical background (medical graduates from Mainland China and medical students). For subjects from whom information could not be directly obtained because of poor cognitive function or illness, the proxy was interviewed instead. For respondents living in the community, the household member responsible for their care was interviewed. For subjects living in institutions, a staff member was used as a proxy.
KEY POINTS

- Health costs of chronic diseases should be estimated using figures for disease burden, taking into account the number developing new disease and the number dying from disease.

- For the Hong Kong Chinese population aged 70 years and over, the disease burden figures were highest for arthritis, hypertension, cardiac disease, and peptic ulcer.

- The estimated economic cost for stroke for the year 2001 is projected to be around US $\$ 250$ million.

- Strategies for preventive efforts can be targeted at diseases with high costs and known preventive methods.

Information was obtained regarding past medical history for the following 10 commonly occurring or chronic medical conditions: arthritis, hypertension, cardiac disease (heart failure or ischaemic heart disease), cerebrovascular disease, chronic obstructive airways disease, peptic ulcer, diabetes mellitus, osteoporotic fracture, malignancy, and dementia. The existence of a condition was based on a doctor's consultation, or regular follow up visits, and use of medication where appropriate.

After an 18 month period, a follow up survey was conducted to determine the development of new diseases, or if death had occurred, the cause of death from the death certificate. The disease burden was calculated as the number of subjects developing new disease plus the number with known disease at the beginning of the 18 month period minus the number who had died during this period. The period of 18 months was chosen for operational reasons, as resource limitations precluded a longer period of follow up.

If one assumes that the incidence rate for these diseases and the mortality for the population in this age group with these diseases remain constant, then the actual period of follow up would not affect the calculation of disease burden. In estimating the economic consequence of a particular disease, stroke was used as an example as the epidemiology of the disease from onset to a 20 month period post ictus had been studied in detail for this

Table 1 Estimation of disease burden in relation to age groups

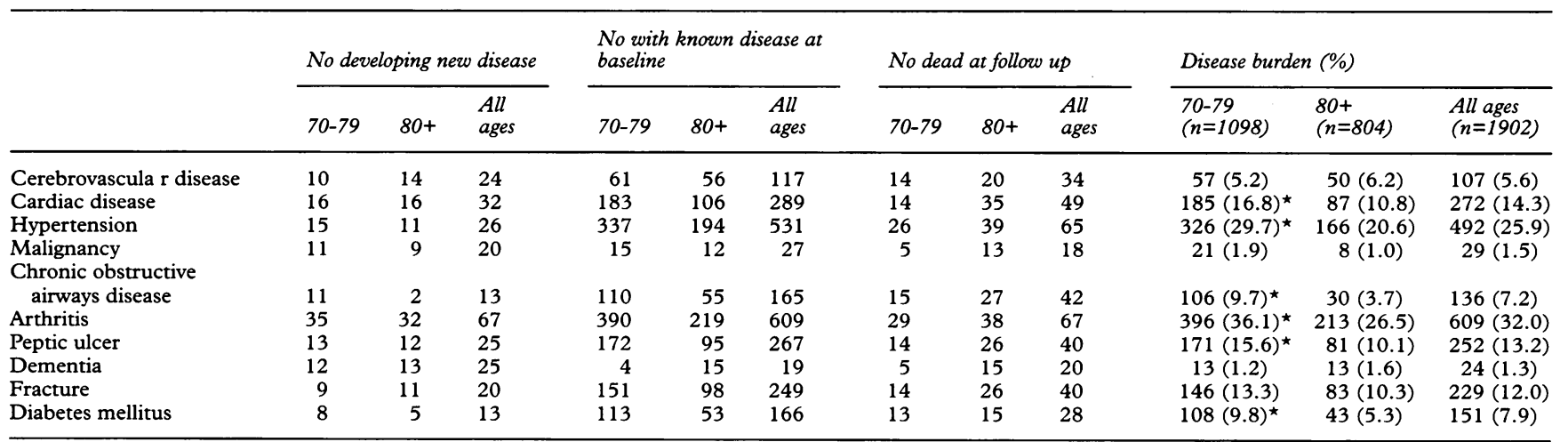

* $70-79$ years compared with $80+$ years, $\chi^{2}$ test $p<0.001$.

Disease burden $=$ number developing new disease + number with known disease - number with the disease who had died. 
Table 2 Disease burden in absolute numbers for the Hong Kong population aged 70 years and over

\begin{tabular}{llcc}
\hline & Burden $\%$ & $\begin{array}{c}\text { Absolute numbers } \\
(1992)^{\star}\end{array}$ & $\begin{array}{c}\text { Projected absolute } \\
\text { number }(2001)^{\star}\end{array}$ \\
\hline Arthritis & 32.0 & 109088 & 145120 \\
Hypertension & 25.9 & 88293 & 117457 \\
Cardiac disease & 14.3 & 48749 & 64851 \\
Peptic ulcer & 13.2 & 44317 & 58955 \\
Old fracture & 12.0 & 40908 & 54420 \\
Diabetes mellitus & 7.9 & 26931 & 35827 \\
Chronic obstructive airways disease & 7.2 & 24545 & 32652 \\
Cerebrovascular disease & 5.6 & 19090 & 25396 \\
Malignancy & 1.5 & 5114 & 6803 \\
Dementia & 1.3 & 4432 & 5896
\end{tabular}

*Total number of population aged 70 years and over $=340900$ in 1992; projected number in 2001 $=453500$.

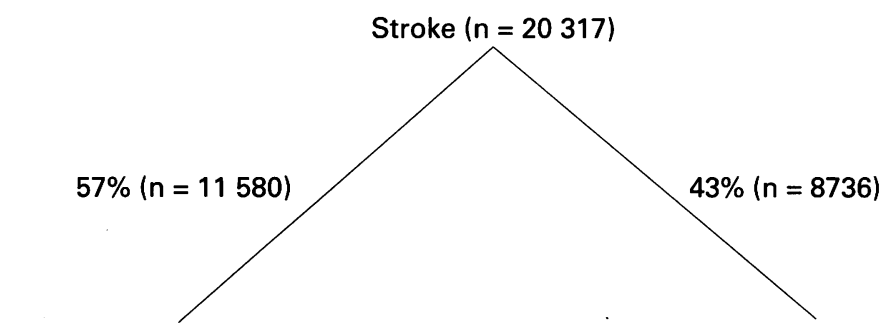

Independent/mild disability

Moderate to severe disability

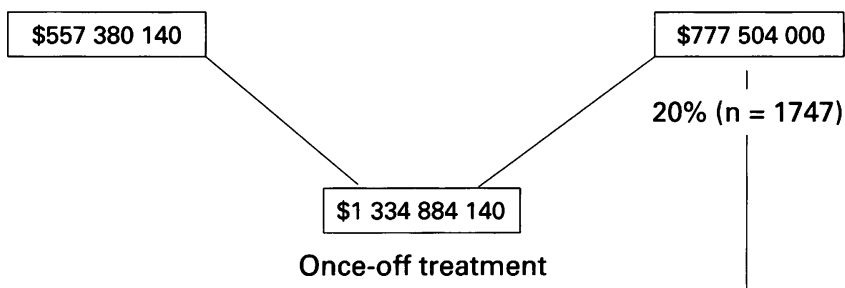

Institutionalised (recurrent cost)

$\$ 8736000$ per month

Figure 1 Outcomes for stroke patients in Hong Kong.

population. ${ }^{78}$ Data are also available regarding costs of different modes of care. ${ }^{910}$

\section{Results}

At baseline, 2032 subjects (999 men, 1033 women) were interviewed. After 18 months, 237 had died and 130 had been lost to follow up. The results are based on 1902 subjects for whom information was complete. Table 1 shows the estimated disease burden for the overall population aged 70 years and over, and also by two age groups : 70-79 years, $80+$ years. The commonest diseases were arthritis, hypertension, cardiac disease, and peptic ulcer. The disease burden was higher among the 70-79 years group compared with the $80+$ years group for six diseases : arthritis, hypertension, cardiac disease, peptic ulcer, diabetes, and chronic obstructive airways disease. The disease burden is expressed in absolute numbers for the Hong Kong elderly population in table 2 . The calculation is based on the census estimates of the number of people in the 70-79 and $80+$ age groups respectively.

To illustrate the use of these data in estimating health care costs resulting from diseases, cerebrovascular disease is used as an example. The outcome of stroke for the Hong Kong Chinese population is shown in figure 1 . The absolute number based on projected popula- tion figures for 2001 is used. It has been estimated that approximately $80 \%$ of patients with acute stroke in Hong Kong are admitted and dealt with by the hospital system, as a result of the poorly developed government primary health care system. The estimated total cost for caring for these patients admitted to hospital in Hong Kong dollars is outlined in rectangles and is calculated as follows:

- Independent/mild disability. Assume 4 days in acute hospital ( $\$ 2000$ per day); 21 days in convalescent hospital ( $\$ 900$ per day); course of therapy at geriatric day hospital (mean cost $\$ 21$ 233). ${ }^{9}$ Cost per patient $=\$ 48133$.

- Moderate to severe disability. Assume 4 days in acute hospital and 3 months in convalescent hospital. Cost per patient $=\$ 89000$.

- Institutional care. Nine per cent of all stroke patients (or $20 \%$ of those who are moderately/ severely disabled) require institutional care. ${ }^{8}$ The average cost of nursing home care is $\$ 5000$ per month.

The economic consequence of a reduction in stroke as a result of preventive methods can also be estimated. For example, if a reduction in the mean population diastolic blood pressure of $7.5 \mathrm{mmHg}$ for two to three years can be achieved, the risk of stroke may be reduced by about $46 \% .{ }^{11}$ This reduction translates into a reduction in cost of approximately HK\$1 billion.

\section{Discussion}

Although elderly people form a lower percentage of the total population in Hong Kong compared with western countries $(8 \%$ aged 65 and over versus $15 \%$ for the UK and $12 \%$ for USA), their number is increasing at a rapid rate, such that by 2003 , the number of people aged 70 years and over is expected to increase by $63 \% .^{12}$ In parallel with this demographic change, health costs have escalated. For example, the increase in expenditure in the hospital sector alone is approximately $18 \%$ per year. ${ }^{13}$ This is partly due to the development of expensive new technology and drugs, but a large component is a result of increasing numbers of elderly with chronic diseases. In any general district hospital, for example, elderly aged 70 years and over occupy at least a third to a half of all acute medical beds. Therefore it is vital to carry out estimations of the economic consequences of this chronic disease burden for the population.

In large population surveys where information regarding the existence of many different medical conditions is required, it is seldom possible to carry out physical examination and investigations for all subjects. The prevalence of diseases could be underestimated, since many diseases in the elderly may remain asymptomatic. ${ }^{14}$ The extent of underestimation is likely to vary between different diseases. Thus, the determination of stroke prevalence by history alone has been shown to have a high sensitivity and specificity, ${ }^{15}$ while for diabetes mellitus, up to half of the patients with the disease may remain undiagnosed. ${ }^{16}$

For some diseases (cardiac and chronic obstructive airways disease, hypertension, dia- 
betes, arthritis, and peptic ulcer), the burden is higher for the 70-79 age group, possibly reflecting the reduced life expectancy of subjects with these diseases. However, the greater extent of underdiagnosis in the $80+$ age group cannot be excluded, since diagnosis through evidence of contact with the health services will underestimate the true prevalence. From an economic view point, it could be argued that underdiagnosis will not affect cost estimations, since no expenditure will be incurred for undiagnosed diseases. For some diseases, the prevalence figures are similar to those for disease burden figures (arthritis, malignancy), while for most of the other diseases, the disease burden figures are lower. This is particularly so for chronic obstructive airways disease. However, for dementia, the disease burden figure is higher than the prevalence figure. These observations probably reflect differences in life expectancies for subjects with different diseases, and illustrate that the use of disease burden estimation would give more accurate estimations of economic consequences.

The use of these figures forms a starting point for calculations of health care costs. These would be different for different diseases which have different patterns of utilisation of expensive technology (such as percutaneous transluminal coronary angioplasty), drugs, AIDS, rehabilitation, and personal care support. As there is comprehensive information regarding stroke and its long term consequences in our population, the economic consequences arising from stroke could be calculated. However, the costs for informal carers of patients have not been taken into account. Similar calculations need to be made for other diseases, since diseases with the highest prevalence may not necessarily be those giving rise to the highest costs. Strategies for preventive efforts can then be made, to focus on diseases with a high cost and known effective prevention.

Funding: this work was supported in part by the Croucher Foundation and the Health Research Fund, Hong Kong. Conflicts of interest: none.

1 Ho SC, Donnan SPB, Sham A. Psychosomatic symptoms, social support and self worth among the elderly in Hong Kong. F Epidemiol Community Health 1988; 42:377-84.

2 Mahoney FI, Barthel DW. Functional evaluation : Barthel index. Med $\mathcal{F} 1965 ; 14: 61-5$.

3 Yesavage JA, Brink TL. Development and validation of a geriatric depression screening scale. A preliminary report. $\mathcal{F}$ geriatric depression screening
Psychiatr Res 1983; 17:37-49.

4 Lee HCB, Chiu HFK, Kwok WY, Leung CM, Kwong PK, Chung DWS. Chinese elderly and the GDS short form : a preliminary study. Clinical Gerontologist 1993; 14(2):37-42.

5 Pattie AH, Gilleard CJ. The Clifton assessment schedule : further validation of a psychogeriatric assessment schedule. Br F Psychiatry 1976; 129:68-72.

6 Rose GA, Blackburn H. Cardiovascular survey methods. Geneva: World Health Organization, 1968

7 Woo J, Kay R, Yuen YK, Nicholls MG. Factors influencing long-term survival and disability among three-month stroke survivors. Neuroepidemiology 1992; 11:143-50.

8 Woo J, Yuen YK, Kay R, Nicholls MG. Survival, disability, and residence twenty months after acute stroke in a Chinese population: implications for community care. Disability and Rehabilitation 1992; 14:36-40.

9 Hui E, Woo J, Or KH, Chu LW, Wong KH. A geriatric day hospital in Hong Kong: an analysis of activities and costs. Disability and Rehabilitation 1995; 17:418-23.

10 Hui E, Lum CM, Woo J, Or KH, Kay RLC. Outcomes of elderly stroke patients. Day hospital versus conventional medical management. Stroke 1995; 26:1616-9.

11 MacMahon S, Peto R, Cutler J, et al. Blood pressure, stroke and coronary heart disease. Lancet 1990; 335:765-74.

12 Hospital Authority. Hospital authority statistical report 1993/ 94. Hong Kong: Hospital Authority 1994; 3 .

13 Hospital Authority. Annual report 1993-1994. Hong Kong: Hospital Authority, 1995.

14 Horan MA. Introduction - presentation of disease in old age. In: Brocklehurst JC, Tallis RC, Fillit HM eds. Textbook of geriatric medicine and gerontology. 4th ed. Edinburgh: Churchill Livingstone, 1992 .

15 O'Mahoney PG, Dobson R, Thomson RG, Rodgers H, James OFW. "Have you ever had a stroke?" is a sensitive
and specific screening question for assessing stroke prevalence. Age Ageing 1995; 24(suppl 2): 10.

16 Woo J, Swaminathan R, Cockram C, et al. The prevalence of diabetes mellitus and an assessment of methods of detection among a community of elderly Chinese in Hong Kong. Diabetologia 1987; 30:863-8. 